\title{
Exchange bias in ferromagnetic/antiferromagnetic bilayers with imperfect interfaces
}

\author{
J Spray and U Nowak \\ Department of Physics, University of York, York YO10 5DD, UK \\ E-mail: un500@york.ac.uk
}

Received 31 July 2006, in final form 12 September 2006

Published 20 October 2006

Online at stacks.iop.org/JPhysD/39/4536

\begin{abstract}
The influence of an imperfect interface on exchange bias (EB) properties is investigated. Within the framework of the domain state model, the EB field $H_{\mathrm{EB}}$ and the coercive field $H_{\mathrm{C}}$ are determined using computer simulations, and they are found to depend strongly on the details of the interface structure. This dependence is sensitive to the dilution of the antiferromagnet (AFM) with non-magnetic defects in the bulk. For the optimal interface structure, giving greatest EB, the optimal dilution is found to be much less than that for an ideal-interface system, taking a value in better agreement with experimental results. Even without any defects in the bulk of the AFM the interface roughness leads to EB for thin antiferromagnetic layers, in accordance with the model by Malozemoff. Finally, the thickness dependence of rough-interface systems is found to differ significantly from that of ideal-interface systems.
\end{abstract}

(Some figures in this article are in colour only in the electronic version)

\section{Introduction}

Exchange bias (EB) is the term used to describe the unidirectional anisotropy found in a ferromagnet (FM) exchanged-coupled to an antiferromagnet (AFM). Although it was first observed around 50 years ago [1], and is widely exploited in magnetic sensor technologies, a full theoretical understanding of the phenomenon remains lacking (for reviews see $[2,3])$.

Recently, the domain state (DS) model was introduced [4], wherein EB is explained by the net AFM interfacial magnetization associated with a DS in the AFM, and variations in EB are explained by the effect of experimental parameters upon this DS. The DS model is supported by experimental [5-8] and computer simulated [9-12] results.

The DS model correctly predicts that some dilution of the AFM bulk-which is conducive to formation of DSs in the AFM-leads to an increased EB. However, prior simulations [9] have predicted an optimal dilution of the order of 50\%, while experiments $[4,7]$ have found it to be of the order of $10 \%$. It is the aim of this work to modify the DS model to include non-planar structure at the interface. As we will show this leads to a new estimate of the optimal dilution which is much closer to that seen in experiment and to new insight regarding the dependence of EB on the details of the interface structure.

\section{Model and method}

Simulations are performed within the framework established by the DS model, which is explained in detail in [9]. Our model is comprised of an Ising AFM of thickness $t_{\mathrm{AFM}}$, coupled to a Heisenberg FM only one monolayer thick. The overall system is a cuboid of size $\left(t_{\mathrm{AFM}}+1\right) \times L \times L$ where $L$ is set to 128 for all the results presented here. Periodic boundary conditions are applied in-plane and open boundary condition out-of-plane. Spins are arranged in a simple cubic lattice. Energies are calculated using the appropriate Ising or Heisenberg Hamiltonians, considering only nearest neighbour exchange interactions and disregarding the dipole interaction.

$$
\begin{aligned}
\mathcal{H}= & -J_{\mathrm{AFM}} \sum_{\langle i, j\rangle \in \mathrm{AFM}} \sigma_{i} \sigma_{j}-\sum_{i \in \mathrm{AFM}} H_{z} \sigma_{i} \\
& -J_{\mathrm{FM}} \sum_{\langle i, j\rangle \in \mathrm{FM}} \vec{S}_{i} \cdot \vec{S}_{j}-\sum_{i \in \mathrm{FM}}\left(d_{z} S_{i_{z}}^{2}+\vec{H} \cdot \vec{S}_{i}\right) \\
& -J_{\mathrm{INT}} \sum_{\langle i \in \mathrm{AFM}, j \in \mathrm{FM}\rangle} \sigma_{i} S_{i_{z}} .
\end{aligned}
$$


The FM spins $\vec{S}_{i}$ couple to each other with an exchange constant $J_{\mathrm{FM}}$, the AFM Ising spins $\sigma_{i}$ to each other by $J_{\mathrm{AFM}}=$ $-1 / 2 J_{\mathrm{FM}}$, and the FM and AFM spins interact with exchange constant $J_{\mathrm{INT}}=-J_{\mathrm{AFM}}$. All other quantities are given in units normalized with respect to these $J$. The FM spins have a uniaxial anisotropy of $d_{z}=0.1 J_{\mathrm{FM}}$, and AFM spins have effectively infinite anisotropy along the same axis. The spins $\sigma$ and $\vec{S}$ are normalized to unity, such that the field variable $\vec{H}$ has units of energy.

For our thermodynamic calculations we use Monte Carlo (MC) methods with the Metropolis algorithm to advance the system, although the results may equally be reproduced using the heat-bath algorithm. Trial steps consist of flips for Ising spins and of random displacements of maximum magnitude 0.1 for Heisenberg spins [13]. An MC step is performed by sweeping across the lattice, performing one trial step for each spin.

The initial cooling procedure is performed over around 20000 MC steps, from $k_{\mathrm{B}} T=J_{\mathrm{FM}}$ to $k_{\mathrm{B}} T=0.1 J_{\mathrm{FM}}$. No applied field is used during cooling but magnetization is provided by the FM layer, which is initially fully magnetized, and remains so since the upper temperature limit is still below the Curie temperature. Each hysteresis loop performed is calculated over around $40000 \mathrm{MC}$ steps.

The bulk of the AFM is randomly diluted with nonmagnetic defects which replace a proportion $p$ of the spins. These defects decrease the energy cost of domain walls in the AFM, facilitating the formation of a DS [9]. As a new aspect in the model, we now introduce some intermixing of the FM and AFM sites in one monolayer at the interface. This intermixing represents a minimum amount of roughness which in a real system will always exist. This mixing is described by a mixing coefficient $R$. In the layer adjacent to the FM monolayer, $R$ is the proportion of spins which are part of the FM rather than part of the AFM. The dilution $p$ continues to act in this layer, such that this layer consists of a proportion $(1-p) R$ of FM sites and $(1-p)(1-R)$ of AFM sites.

Note, that for $R \rightarrow 0$ and $R \rightarrow 1$ the interface is perfectly flat and compensated while the roughness is maximal for $R=0.5$. The model presented here is clearly a vast simplification of a real interface, which usually will have larger roughness with more than one monolayer involved, but as we will show in the following the results it produces are nevertheless intriguing.

\section{Results}

The effect of the interface mixing $R$ upon the EB field $H_{\mathrm{EB}}$ is shown in figure 1 . We see that systems with low or no dilution feature a strong response in EB to the introduction of roughness, while systems with high dilution that previously [9] gave maximal EB are less affected. Comparing these results to previous work [9], we note two most important features: firstly, that EB is exhibited even for systems with no dilution whatever ( $p=0)$ and, secondly, that the rough interface renders a greater $\mathrm{EB}\left(H_{\mathrm{EB}} \approx 0.027 J_{\mathrm{INT}}\right)$ than the greatest previous result from the DS model $\left(H_{\mathrm{EB}} \approx 0.02 J_{\mathrm{INT}}\right)$. Interestingly, the maximal $\mathrm{EB}$ is achieved for $R \approx 0.2$, i.e. for systems with a small amount of FM spins in the interface layer of the AFM.

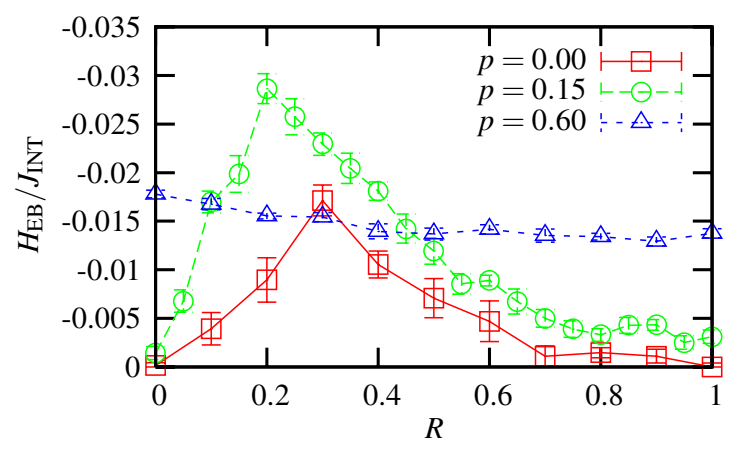

Figure 1. Variation of the EB field $H_{\mathrm{EB}}$ with the interface mixing $R$ for different degrees of AFM dilution $p$. The thickness of the AFM is $t_{\mathrm{AFM}}=4$ monolayers.

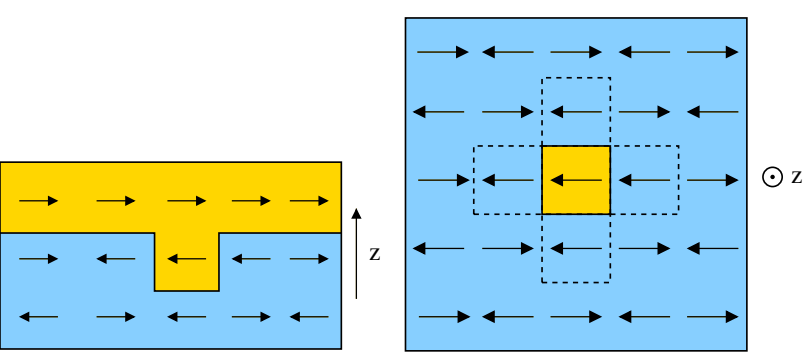

Figure 2. Isolated FM spin in the interface layer of the AFM as it is typical for low $R$. The sketch on the left-hand side is a cross-section while the right-hand side shows a top view of the interface. On the left-hand side the FM is reversed while the lone FM spin embedded in the AFM is pinned by the interaction with its AFM neighbours.

The reason for the enhanced EB lies in the optimized interface coupling associated with low values of $R$. For a given DS, EB is greater in slightly rough systems because it effectively enhances the FM-AFM coupling. To understand this, consider an FM spin embedded in the AFM (as sketched in figure 2), having 5 AFM neighbours and 1 FM neighbour. This lone FM spin will be pinned by the AFM, since it is $5\left|J_{\mathrm{AFM}}\right|>\left|J_{\mathrm{FM}}\right|$. For positive interface exchange interaction it acts like a reversed AFM spin, except that unlike a normal AFM spin it has a ferromagnetic interaction with its FM neighbour, thus increasing the net exchange coupling between FM and AFM since in usual EB systems the ferromagnetic interactions are the strongest interactions. Note that the above argument works only in the limit of small $R$ where one finds mainly isolated FM spins embedded in the AFM, instead of larger groups of FM spins or even lone AFM spins in an FM environment. This fact explains the asymmetry of the curves in figure 1 which one might naively not expect. The largest EB field is achieved for a rather small amount of FM spins in the mixed interface layer. However, it is not obvious how the interface mixing could be controlled microscopically in an experiment.

The peak in $H_{\mathrm{EB}}$ with respect to $R$ results from the tendency of excessive $R$ to destroy the enhanced interface coupling described above: if there are too many FM spins in the interface layer then they form groups which are no longer frozen into the AFM. For example, for $R=0.5$ an FM interface-layer spin has on average 3 FM neighbours and 3 AFM neighbours, so with $J_{\mathrm{FM}}>\left|J_{\mathrm{AFM}}\right|$ it follows the FM during hysteresis rather than being pinned by the 


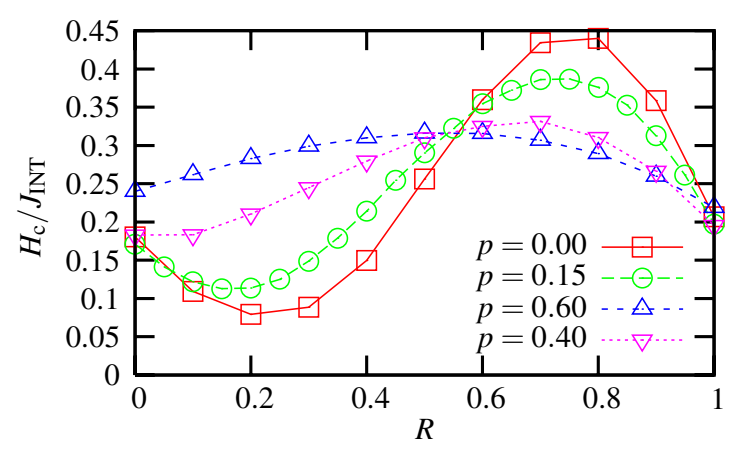

Figure 3. Variation of the coercive field $H_{\mathrm{C}}$ with $R$, for various dilutions.

AFM. Furthermore, the peak moves left with dilution because replacing some AFM sites with defects reduces the $R$ threshold required to allow FM spins mixed into the AFM to move with the FM rather than being pinned to the AFM.

The presence of DSs fostering EB even at $p=0$ may be explained by the defect-like domain-wall-pinning action of FM spins in the AFM interface layer. For a smooth interface it was found that having defects only at the interface was not sufficient to yield an EB, but the use of FM spins conveys an advantage over defects since they enhance rather than diminishing the interface coupling. This heightened interface coupling increases both, the exchange field provided by the FM during cooling and the action of the AFM upon the FM during hysteresis. Note, that in the limit of vanishing bulk dilution our model coincides with the model of Malozemoff [14-16] for minimal roughness so that our findings qualitatively support his earlier ideas. To the best of our knowledge, the simulations presented here are the first numerical investigations of Malozemoff's model.

For highly diluted ( $p \approx 0.6$ ) systems $R$ appears to have only little effect. To understand this, consider the case $p=0.5$. Here, FM interface spins have on average 1 FM neighbour, 2.5 defect neighbours and 2.5 AFM neighbours. They are no longer so firmly tied to the AFM, which annuls the enhanced interface coupling. For such high dilutions, roughness does not change the interface structure so much. Furthermore, because the DS is already well formed due to dilution, the roughness does not improve the AFM domain structure either. Overall, for $p=0.6$ we see a slight decrease in EB with $R$ as can be explained by the increasing thickness of the FM. The values of $H_{\mathrm{EB}}$ for $R=1$ are in general about the same as those for $R=0$. This is quite unexpected since the EB should decrease with thickness of the FM proportional to $1 / t_{\mathrm{FM}}$. However, within the error bars it does not decrease at all. Probably, this is an artefact of the small AFM thicknesses used $\left(t_{\mathrm{AFM}}=4\right)$ since the net decrease in the thickness of the AFM with $R$ increases its magnetization due to the lower energy cost of domain formation.

Maximal EB is found in systems with a small dilution ( $p \approx 0.10$ ), as well as some mixing $(R \approx 0.2)$ at the interface. This is an important result since it agrees with the experimental fact that greatest EB is found in systems with dilutions of the order of $10 \%[4,7]$ while previous simulations of the DS model agreed with the general trend in $H_{\mathrm{EB}}$ versus $p$ but massively overestimated the optimal dilution $[4,9]$.
Figure 3 shows the response of the coercive field $H_{\mathrm{C}}$ to the introduction of the mixed interface. For lower dilutions ( $p=0.0, p=0.15$ ), small $R$ values lead to a decrease in $H_{\mathrm{C}}$ as compared with its smooth-interface value while for higher values of $R$ it is seen to increase. For higher values of the bulk dilution though, the coercive field is always enhanced by the interface roughness. This result provides some insight into the behaviour of the exchange interactions at the interface.

For an explanation consider two extreme cases: on the one hand that of very low $R$, where single FM spins are embedded in an AFM-dominated interface layer, and on the other hand that of high $R$, where single AFM spins are embedded in an FM-dominated interface layer. In the first case, as discussed above and illustrated in figure 2, the isolated FM spins are pinned by the AFM. These spins will be pinned in both the possible directions. The imbalance of the number of spins pinned either in the initial directions of the FM or in the opposite direction gives rise to EB. However, those spins pinned in the direction opposite to the FM (on both sides of the loop) act as nucleation sites, leading to reversal at lower $H_{\mathrm{C}}$. In the second case, the isolated AFM spins have $5 \mathrm{FM}$ neighbours and 1 AFM neighbour. The interaction with the AFM neighbour may be disregarded since its magnitude is much weaker so that upon reversal of the FM these AFM spins will flip. However, due to their large anisotropy these spins act like Ising (highly anisotropic) FM spins, albeit with only $J_{\text {INT }}$ exchange coupling to their neighbours in the FM. This induces an effective uniaxial anisotropy in the FM and thus increases $H_{\mathrm{C}}$.

Furthermore, we notice that both the effects above, the low- $R$ decrease in $H_{\mathrm{C}}$ as well as its high- $R$ increase, are diminished with greater dilution. The first one is counteracted by dilution because the formation of groups of a single FM spins surrounded by AFM spins becomes less probable. The second one is counteracted by dilution because AFM spins in the interface layer have on average fewer FM neighbours, thus decreasing the overall coupling of the anisotropic AFM spins into the FM and, hence, inducing less anisotropy.

Figure 4 shows the effect of $R$ upon the hysteresis loops of the FM. For a value of $R=0.25$ which induces a large EB the loop is narrower and less squared. Larger values such as $R=0.75$ render the loop wider than the $R=0$ case does, but retain about the same degree of squareness. This shows that the $R=0.25$ system features a more gradual reversal of the FM, indicating a broader distribution of switching fields. As already mentioned above, for low values of $R$ the random mixing leads to single FM spins pinned by the AFM with very high switching field. Regions in the FM adjacent to these magnetized spots of the interface layer will either act as nucleation centres or as pinned regions depending on whether the direction of the magnetization in the interface layer agrees with the direction of the external field or not. This mechanism leads to the observed broadening of the hysteresis loop. The associated narrowing of the loop-as well as the widening for larger values of $R-$ was already explained above in connection with the coercive fields. We also note that the low- $R$ loop does not come as close to saturation as the other loops. This shows once again that some FM spins embedded in the interface layer are pinned by the AFM.

As previously seen in simulations of the DS model [17], the strength of EB is strongly linked to the thickness $t_{\mathrm{AFM}}$ of 


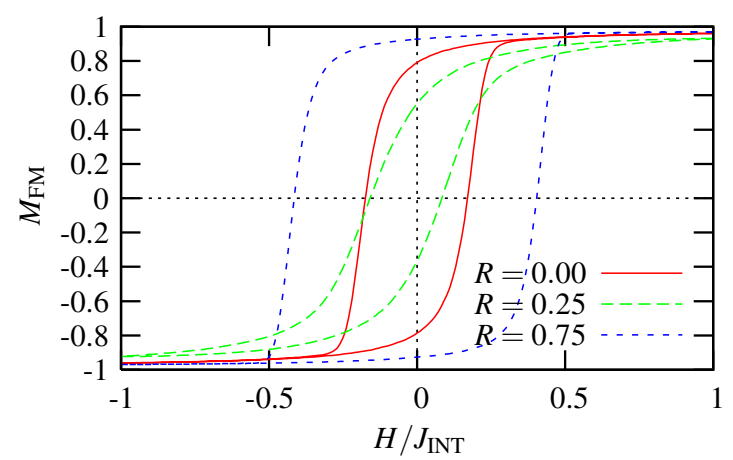

Figure 4. Hysteresis loops of the FM for different values of the interface mixing $R(p=0.1)$.

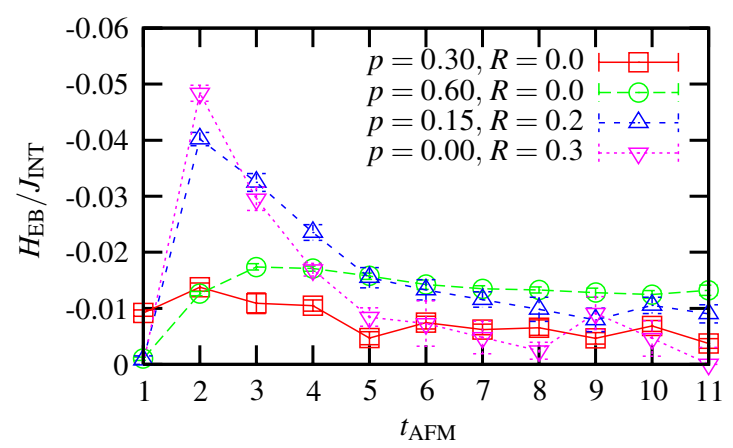

Figure 5. Thickness dependence of the EB field for different values of bulk dilution and interface mixing.

the AFM. In general, a larger thickness of the AFM enhances its stability which is especially important for the EB at higher temperatures. On the other hand it hinders the creation of a $\mathrm{DS}$, which is the origin of $\mathrm{EB}$, since the domain wall energy increases with AFM thickness as long as the domain walls are mainly perpendicular to the interface. The combination of these two effects leads often to a maximum of the EB for a certain thickness. As shown in figure 5, in general these findings are reproduced here. Nevertheless, the thickness dependence of systems with mixed interfaces display also some distinct features.

Those systems with interface mixing show a very high peak for $t_{\mathrm{AFM}}=2$, decreasing for greater thicknesses. Both undiluted $(p=0.0)$ and slightly diluted $(p=0.15)$ systems are shown. As before we see a general enhancement in EB for a slightly diluted system over an undiluted system. The undiluted rough system, however, gives the greater EB for extremely small thicknesses $\left(t_{\mathrm{AFM}}=2\right)$. This may be attributed to the more optimal interface coupling in the absence of dilution, overriding the bulk-stabilization effects of defects since the system is so shallow as to have practically no bulk.

The EB borne of the rough interfaces diminishes for thicker systems, eventually falling below that given by the highly diluted smooth interface system $(p=0.6)$. This is caused by the fact that without (or with only small) bulk dilution domain walls in the bulk of the system cost more energy so that the DS is suppressed with increasing thickness. However, the error bars for rough, undiluted systems become large at $t_{\mathrm{AFM}} \geqslant 6$ due to finite size effects. Indeed, visualization of the AFM DSs shows that these thick undiluted systems tend to form large cylindrical domains finally having diameters of the order of the system size. The question whether a surface roughness alone-without any defects in the bulk of the AFM-can lead to an EB in the limit of large AFM thickness cannot be answered. However, for sufficiently small AFM thicknesses the original ideas by Malozemoff [14-16] are qualitatively confirmed by our simulations.

\section{Summary}

Investigating the influence of interface mixing on $\mathrm{EB}$ properties we find that the EB field $H_{\mathrm{EB}}$ strongly depends on the details of the interface structure. A smaller amount of FM spins in the topmost AFM monolayer enhances EB, while a larger amount of FM spins in this layer leads to a lower EB effect. For the optimal amount of interface mixing, giving greatest EB, the optimal dilution of the bulk of the AFM is found to be much less than that for an ideal-interface system, taking a value in good agreement with experimental results. In the limit of low AFM thickness even without any defects in the bulk of the AFM the interface roughness leads to EB in accordance with the model by Malozemoff.

\section{Acknowledgment}

The use of the White Rose Grid computational resources at the University of Sheffield is gratefully acknowledged.

\section{References}

[1] Meiklejohn W H and Bean C P 1956 Phys. Rev. 1021413

[2] Nogués J and Schuller I K 1999 J. Magn. Magn. Mater. 192203

[3] Stamps R L 2000 J. Phys. D: Appl. Phys. 33 R247

[4] Miltényi P, Gierlings M, Keller J, Beschoten B, Güntherodt G, Nowak U and Usadel K D 2000 Phys. Rev. Lett. 844224

[5] Mewes T, Lopusnik R, Fassbender J, Hillebrands B, Jung M, Engel D, Ehresmann A and Schmoranzer H 2000 Appl. Phys. Lett. 761057

[6] Mougin A, Mewes T, Jung M, Engel D, Ehresmann A, Schmoranzer H, Fassbender J and Hillebrands B 2001 Phys. Rev. В 6360409

[7] Keller J, Miltényi P, Beschoten B, Güntherodt G, Nowak U and Usadel K D 2002 Phys. Rev. B 6614431

[8] Shi H T, Lederman D and Fullerton E E C 2002 J. Appl. Phys. 917763

[9] Nowak U, Usadel K D, Miltényi P, Keller J, Beschoten B and Güntherodt G 2002 Phys. Rev. B 6614430

[10] Nowak U, Misra A and Usadel K D 2002 J. Magn. Magn. Mater. 240243

[11] Beckmann B, Nowak U and Usadel K D 2003 Phys. Rev. Lett. 91187201

[12] Scholten G, Usadel K D and Nowak U 2005 Phys. Rev. B 7164413

[13] Nowak U 2001 Annual Reviews of Computational Physics IX ed D Stauffer (Singapore: World Scientific) p 105

[14] Malozemoff A P 1987 Phys. Rev. B 353679

[15] Malozemoff A P 1988 J. Appl. Phys. 633874

[16] Malozemoff A P 1988 Phys. Rev. B 377673

[17] Nowak U, Misra A and Usadel K D 2001 J. Appl. Phys. 897269 